\title{
On impedance in shock-refraction problems
}

\author{
A. Haselbacher
}

Received: 30 January 2012 / Revised: 20 April 2012 / Accepted: 3 May 2012 / Published online: 22 May 2012

(c) Springer-Verlag 2012

\begin{abstract}
Publications on shock-refraction problems typically predict wave patterns resulting from the interaction from the acoustic-impedance ratio. In this note, an analysis based on the shock-impedance ratio is used to derive conditions under which the acoustic-impedance ratio predicts the incorrect type of reflected wave. The range of density ratios for which incorrect types of reflected waves are predicted is found to be quite narrow.
\end{abstract}

Keywords Shock-wave refraction - Shock impedance . Shock-bubble interaction

\section{Motivation}

The propagation of shock waves through inhomogeneous gases is an interesting and important problem that has been considered by many authors. In its simplest form, the problem consists of a planar shock wave propagating in a perfect gas of uniform density $\rho_{1}$ and pressure $p_{1}$ toward a single spherical inhomogeneity containing a perfect gas of density $\rho_{2}$ and pressure $p_{2}=p_{1}$. The shock wave is refracted and diffracted on interacting with the inhomogeneity, leading to complicated wave patterns and large distortions of the inhomogeneity.

\section{Communicated by R. Bonazza.}

A. Haselbacher

Department of Mechanical and Aerospace Engineering,

University of Florida, Gainesville, FL 32611-6300, USA

Present Address:

A. Haselbacher $(\varangle)$

Institute of Energy Technology, Department of Mechanical and Process Engineering, ETH Zürich, Sonneggstr. 3, 8092 Zurich, Switzerland

e-mail: haselbac@ethz.ch
Publications on shock-refraction problems typically use the ratio $Z_{2} / Z_{1}$, where $Z_{i}=\rho_{i} c_{i}$ is the acoustic impedance and $c$ is the speed of sound, to determine the wave pattern (see Niederhaus et al. [1] and Ranjan et al. [2] for recent examples). It is stated that the reflected wave is a shock wave if $Z_{2} / Z_{1}>1$ and that the reflected wave is an expansion wave if $Z_{2} / Z_{1}<1$.

However, the acoustic-impedance ratio is applicable only in the limit of an infinitely weak shock wave. For a shock wave of finite strength, the shock-wave impedance $Z_{i j}^{s}$ must be used instead. Following Henderson [3], the shock-wave impedance is defined as

$$
Z_{i j}^{s}=\rho_{i} c_{i} \sqrt{1+\frac{\gamma+1}{2 \gamma}\left(p_{j i}-1\right)}=Z_{i} M_{s}=\rho_{i} u_{\mathrm{s}}
$$

where the subscripts $i$ and $j$ denote the states ahead of and behind the shock wave, $\gamma$ is the ratio of specific heats, $p_{j i}=$ $p_{j} / p_{i}, M_{s}$ is the Mach number of the shock wave, and $u_{\mathrm{s}}$ is the speed of the shock wave. In the limit of an infinitely weak shock wave, $Z_{i j}^{s} \rightarrow Z_{i}$.

The objective of this note is to derive the conditions under which the use of the acoustic instead of the shock-wave impedance leads to incorrect prediction of the type of reflected wave. The analysis is based on the assumption of one-dimensional flow, see Fig. 1, where it is noted that the density inhomogeneity now occupies region 5 . The results thus apply only to the very early stages of the interaction between the shock wave and the spherical inhomogeneity. More elaborate theories can be derived, but these theories are typically very complex. For example, Henderson [4] analyzed the interaction of a plane shock wave with a planar, but non-aligned, inhomogeneity. At first sight, this problem appears to be only marginally more complex, but it leads to 


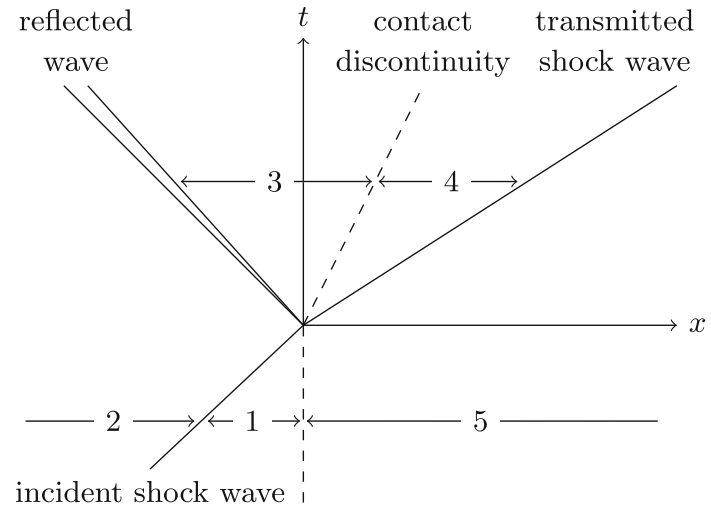

Fig. 1 Schematic illustration shock-refraction problem in the $x-t$ plane to indicate notation used. Because this is only a schematic illustration, the slopes of the lines should not be interpreted as implying restrictions on the speeds of the incident and transmitted shock waves

a polynomial equation of degree 12 and exhibits regular and irregular refraction patterns.

\section{Analysis}

It is useful to briefly review the standard theory developed by Taub [5] and Paterson [6] because it serves as the foundation for the subsequent analysis, see also Glass and Sislian [7]. The theory states that the reflected wave is a shock or expansion wave depending on whether the ratio of impedances of the transmitted and incident shock waves is greater or smaller than unity,

$\frac{Z_{54}^{s}}{Z_{12}^{s}}=\frac{\rho_{5} c_{5}}{\rho_{1} c_{1}} \sqrt{\frac{1+\frac{\gamma_{5}+1}{2 \gamma_{5}}\left(p_{45}-1\right)}{1+\frac{\gamma_{1}+1}{2 \gamma_{1}}\left(p_{21}-1\right)}} \gtrless 1$.

Equality of pressure across the contact discontinuity gives $p_{45}=p_{31}=p_{21} p_{32}$. Following [6], examining the problem in the $(p, u)$ plane indicates that the impedance ratio can be evaluated for $p_{32}=1$. Then (2) can be expressed as

$D\left(p_{21}-1\right) \gtrless N$,

where

$D=\frac{\gamma_{5}+1}{2 \gamma_{5}}-\frac{\gamma_{1}+1}{2 \gamma_{1}} \frac{\gamma_{1} \rho_{1}}{\gamma_{5} \rho_{5}}$

$N=\frac{\gamma_{1} \rho_{1}}{\gamma_{5} \rho_{5}}-1$.

Four cases need to be considered.

1. $\quad D>0$ and $N>0$, so $\left(\gamma_{1}+1\right) /\left(\gamma_{5}+1\right)<\rho_{5} / \rho_{1}<$ $\gamma_{1} / \gamma_{5}$. The inequalities can be satisfied only if $\gamma_{5}<\gamma_{1}$. The reflected wave can be a shock wave or an expansion
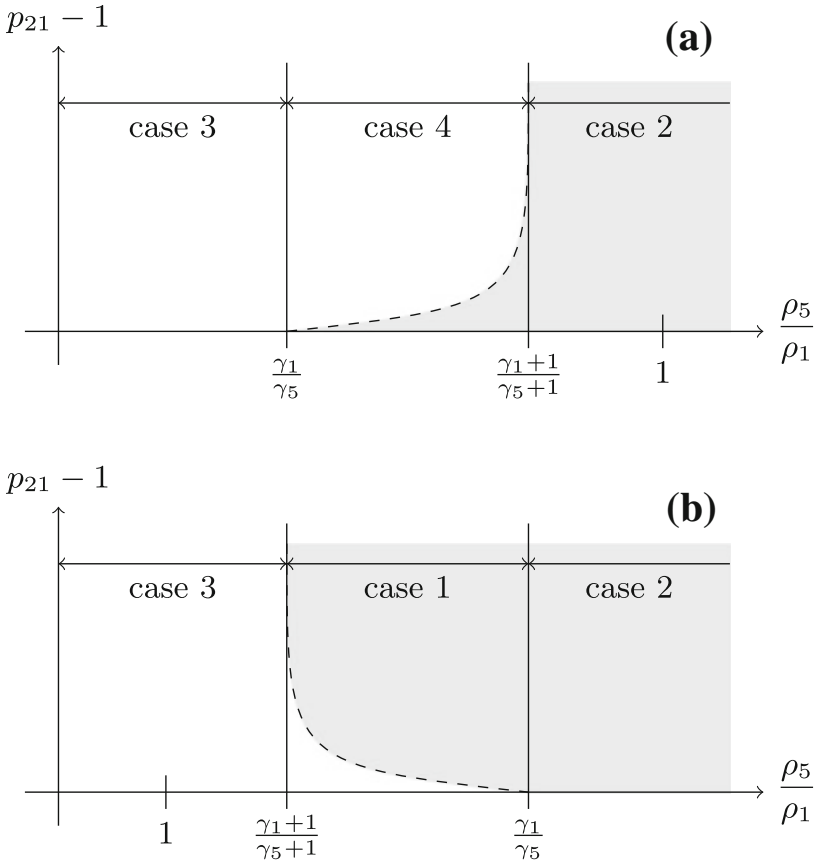

Fig. 2 Schematic illustration of types of reflected waves as determined by shock-impedance ratio as a function of $\rho_{5} / \rho_{1}, \gamma_{1}, \gamma_{5}$, and $p_{21}$ for $\mathbf{a}$ $\gamma_{1}<\gamma_{5}$ and $\mathbf{b} \gamma_{1}>\gamma_{5}$. The shaded regions indicate that the reflected wave is a shock wave. The lack of shading indicates that the reflected wave is an expansion wave. The dashed lines are given by (6) and (7)

wave depending on

$$
p_{21}-1 \gtrless\left(\frac{\gamma_{1} \rho_{1}}{\gamma_{5} \rho_{5}}-1\right) /\left(\frac{\gamma_{5}+1}{2 \gamma_{5}}-\frac{\gamma_{1}+1}{2 \gamma_{1}} \frac{\gamma_{1} \rho_{1}}{\gamma_{5} \rho_{5}}\right) .
$$

2. $D>0$ and $N<0$, so $\rho_{5} / \rho_{1}>\left(\gamma_{1}+1\right) /\left(\gamma_{5}+1\right)$ if $\gamma_{5}>\gamma_{1}$ and $\rho_{5} / \rho_{1}>\gamma_{1} / \gamma_{5}$ if $\gamma_{5}<\gamma_{1}$. The reflected wave is a shock wave.

3. $D<0$ and $N>0$, so $\rho_{5} / \rho_{1}<\gamma_{1} / \gamma_{5}$ if $\gamma_{5}>\gamma_{1}$ and $\rho_{5} / \rho_{1}<\left(\gamma_{1}+1\right) /\left(\gamma_{5}+1\right)$ if $\gamma_{5}<\gamma_{1}$. The reflected wave is an expansion wave.

4. $\quad D<0$ and $N<0$, so $\gamma_{1} / \gamma_{5}<\rho_{5} / \rho_{1}<\left(\gamma_{1}+1\right) /\left(\gamma_{5}+\right.$ $1)$. The inequalities can be satisfied only if $\gamma_{5}>\gamma_{1}$. The reflected wave can be a shock wave or expansion wave depending on

$$
p_{21}-1 \lessgtr\left(\frac{\gamma_{1} \rho_{1}}{\gamma_{5} \rho_{5}}-1\right) /\left(\frac{\gamma_{5}+1}{2 \gamma_{5}}-\frac{\gamma_{1}+1}{2 \gamma_{1}} \frac{\gamma_{1} \rho_{1}}{\gamma_{5} \rho_{5}}\right) .
$$

The results of this analysis are depicted schematically in Fig. 2.

We now turn our attention to determine when the type of reflected wave is different when determined from the acoustic-impedance ratio compared to the shock-impedance ratio. 
To this end, we write (1) as

$\frac{Z_{54}^{s}}{Z_{12}^{s}}=\frac{Z_{5}}{Z_{1}} \frac{M_{s, t}}{M_{s, i}} \gtrless 1$,

where $M_{s, t}$ and $M_{s, i}$ are the Mach numbers of the transmitted and incident shock waves, respectively. We need to consider two main cases.

1. $Z_{5} / Z_{1}<1$ and $M_{s, t} / M_{s, i}>Z_{1} / Z_{5}$ (the acoustic-impedance ratio incorrectly predicts a reflected expansion wave). Then

$\frac{\gamma_{1}\left[\left(\gamma_{5}+1\right) p_{21}+\left(\gamma_{5}-1\right)\right]}{\gamma_{5}\left[\left(\gamma_{1}+1\right) p_{21}+\left(\gamma_{1}-1\right)\right]}>\frac{\gamma_{1} \rho_{1}}{\gamma_{5} \rho_{5}}>1$.

From the left inequality we obtain $D\left(p_{21}-1\right)>N$, where $D$ and $N$ are given by (4) and (5). The right inequality implies that $N>0$ or

$\frac{\rho_{5}}{\rho_{1}}<\frac{\gamma_{1}}{\gamma_{5}}$

We need to consider two subcases. If we assume that $D>0$, then

$\frac{\rho_{5}}{\rho_{1}}>\frac{\gamma_{1}+1}{\gamma_{5}+1}$.

The bounds on $\rho_{5} / \rho_{1}$ are related through

$$
\begin{aligned}
& \frac{\gamma_{1}+1}{\gamma_{5}+1}<\frac{\gamma_{1}}{\gamma_{5}} \quad \text { if } \quad \gamma_{1}>\gamma_{5}, \\
& \frac{\gamma_{1}}{\gamma_{5}}<\frac{\gamma_{1}+1}{\gamma_{5}+1} \quad \text { if } \quad \gamma_{1}<\gamma_{5} .
\end{aligned}
$$

It is impossible to satisfy simultaneously (10), (11), and (13). We are left with

$\frac{\gamma_{1}+1}{\gamma_{5}+1}<\frac{\rho_{5}}{\rho_{1}}<\frac{\gamma_{1}}{\gamma_{5}}$ if $\frac{\gamma_{1}}{\gamma_{5}}>1$

and

$p_{21}-1>\left(\frac{\gamma_{1} \rho_{1}}{\gamma_{5} \rho_{5}}-1\right) /\left(\frac{\gamma_{5}+1}{2 \gamma_{5}}-\frac{\gamma_{1}+1}{2 \gamma_{1}} \frac{\gamma_{1} \rho_{1}}{\gamma_{5} \rho_{5}}\right)$.

On the other hand, if we assume that $D<0$, then $p_{21}-$ $1<N / D<0$, which cannot be satisfied by any $p_{21}>1$.

2. $Z_{5} / Z_{1}>1$ and $M_{s, t} / M_{s, i}<Z_{1} / Z_{5}$ (the acousticimpedance ratio incorrectly predicts a reflected shock wave). Then

$\frac{\gamma_{1}\left[\left(\gamma_{5}+1\right) p_{21}+\left(\gamma_{5}-1\right)\right]}{\gamma_{5}\left[\left(\gamma_{1}+1\right) p_{21}+\left(\gamma_{1}-1\right)\right]}<\frac{\gamma_{1} \rho_{1}}{\gamma_{5} \rho_{5}}<1$.

From the left inequality we obtain $D\left(p_{21}-1\right)<N$, where $D$ and $N$ are defined in (4) and (5). The right inequality implies that $N<0$ or

$\frac{\rho_{5}}{\rho_{1}}>\frac{\gamma_{1}}{\gamma_{5}}$

Again we need to consider two subcases. If we assume that $D>0$, then $p_{21}-1<N / D<0$, which cannot be satisfied by any $p_{21}>1$. On the other hand, if we assume that $D<0$, then

$\frac{\rho_{5}}{\rho_{1}}<\frac{\gamma_{1}+1}{\gamma_{5}+1}$

The lower bound given by (17) and the upper bound given by (18) are related through (12) and (13). No value of $\rho_{5} / \rho_{1}$ that satisfies (17) and (18) can satisfy (12). Thus we are left with

$\frac{\gamma_{1}}{\gamma_{5}}<\frac{\rho_{5}}{\rho_{1}}<\frac{\gamma_{1}+1}{\gamma_{5}+1}$ if $\frac{\gamma_{1}}{\gamma_{5}}<1$

and $p_{21}$ is again given by (15).

These results are summarized in Fig. 3. By comparing with Fig.2, we see that the use of the acoustic-impedance ratio leads to incorrect predictions for cases $(a)$ and $(d)$ of the standard theory in which the shock-impedance ratio predicts either a reflected shock wave or expansion wave depending on the pressure ratio of the incident shock wave.

\section{Discussion}

Having derived the conditions under which the acousticimpedance ratio predicts the incorrect type of reflected wave in shock-refraction problems, it remains to be established how restrictive the conditions are. Defining

$\Gamma=\frac{\gamma_{5}}{\gamma_{1}}$

the conditions relate $\gamma_{1}, \Gamma, \rho_{5} / \rho_{1}$, and $p_{21}$. Because $1<\gamma \leq$ $5 / 3$, we have $3 / 5<\Gamma \leq 5 / 3$. For $\gamma_{1}=1.4$, the restrictions can be represented by Fig. 4 . The plot is to be interpreted in the following manner. The acoustic-impedance ratio predicts the incorrect type of reflected wave if the point $\left(\Gamma, \rho_{5} / \rho_{1}\right)$ lies within the regions bounded by the (dashed) lines $\rho_{5} / \rho_{1}=1 / \Gamma$ 

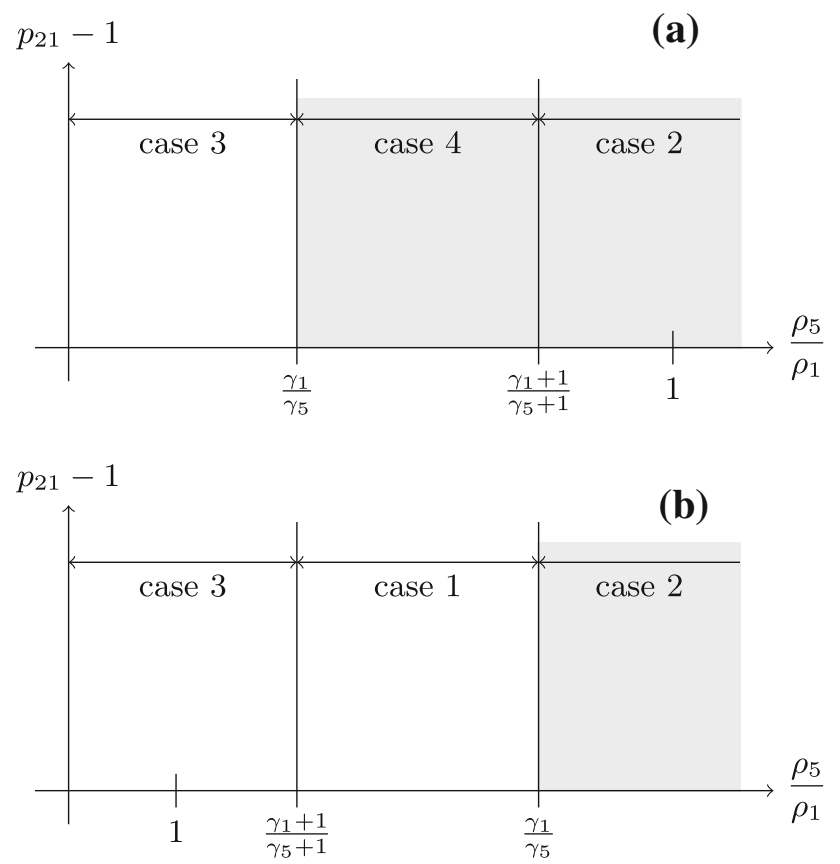

Fig. 3 Schematic illustration of types of reflected waves as determined by acoustic-impedance ratio as a function of $\rho_{5} / \rho_{1}, \gamma_{1}$, and $\gamma_{5}$ for a $\gamma_{1}<\gamma_{5}$ and $\mathbf{b} \gamma_{1}>\gamma_{5}$. The shaded regions indicate that the reflected wave is a shock wave. The lack of shading indicates that the reflected wave is an expansion wave

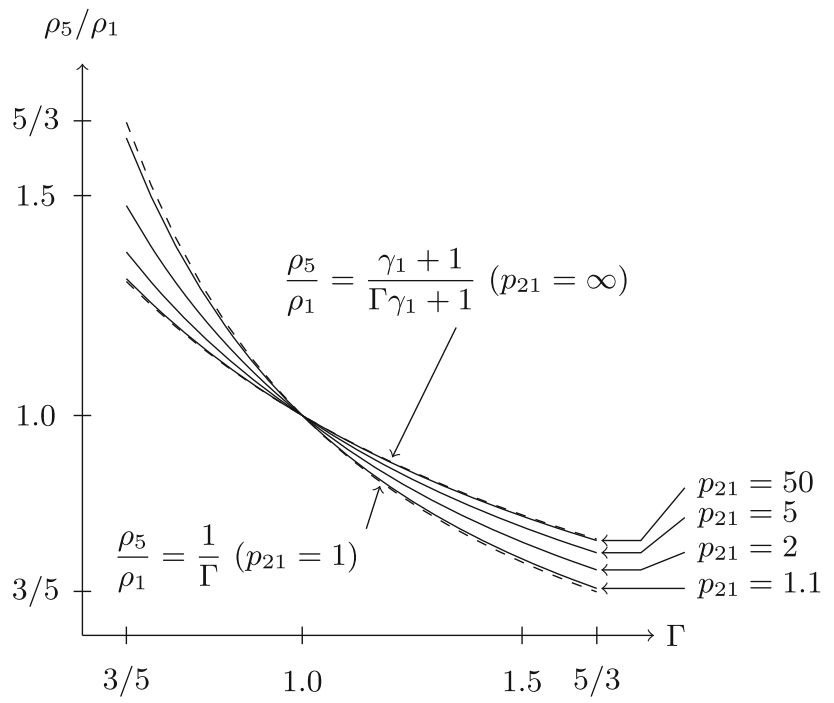

Fig. 4 Values of $p_{21}$ above which the acoustic-impedance ratio predicts the incorrect type of reflected wave as a function of $\Gamma$ and $\rho_{5} / \rho_{1}$ for $\gamma_{1}=1.4$

and $\rho_{5} / \rho_{1}=\left(\gamma_{1}+1\right) /\left(\Gamma \gamma_{1}+1\right)$ and if $p_{21}$ exceeds the values shown in the figure.

It is seen that the range of $\rho_{5} / \rho_{1}$ over which incorrect types of reflected waves are predicted is $O(1)$ and quite narrow. For obvious reasons, shock-refraction experiments usually consider density ratios that are significantly different from unity.
As a result, none of the articles published on shock-refraction problems appears to include an experiment in which the acoustic-impedance ratio predicts the incorrect type of reflected wave. This may explain why the applicability of the acoustic-impedance ratio to shock-refraction problems has not been investigated before. Nevertheless, the conditions derived here may be of use for future studies.

It should be noted that along the line $\rho_{5} / \rho_{1}=\left(\gamma_{1}+\right.$ $1) /\left(\Gamma \gamma_{1}+1\right)$, we have $D=0$ and hence $p_{21}=\infty$ (unless $\Gamma=1$, a case that is of no interest). At high values of $p_{21}$, i.e., for strong shock waves, of course, the assumption of perfect-gas behavior becomes questionable. Data presented by Owczarek [8, section 5.4] indicate that real-gas effects on the pressure ratio across a shock wave in air are negligible provided that $M_{S} \lesssim 8\left(p_{21} \lesssim 75\right)$ if the state ahead of the shock wave is at standard conditions. As may be seen from Fig. 4, curves corresponding to such large values of $p_{21}$ are essentially indistinguishable from the curve for $p_{21}=\infty$. For this reason, and because shock-refraction experiments are usually carried out at conditions that ensure perfect-gas behavior, Fig. 4 should be useful for a wide range of conditions.

\section{References}

1. Niederhaus, J.H., Greenough, J.A., Oakley, J.G., Ranjan, D., Anderson, M.H., Bonazza, R.: A computational parameter study for the three-dimensional shock-bubble interaction. J. Fluid Mech. 594, 85-124 (2008)

2. Ranjan, D., Oakley, J., Bonazza, R.: Shock-bubble interactions. Annu. Rev. Fluid Mech. 43, 117-140 (2011)

3. Henderson, L.F.: On the refraction of shock waves. J. Fluid Mech. 198, 365-386 (1989)

4. Henderson, L.F.: The refraction of a plane shock wave at a gas interface. J. Fluid Mech. 26, 607-637 (1966)

5. Taub, A.H.: Refraction of Plane Shock Waves. Phys. Rev. 72(1), 51-60 (1947)

6. Paterson, S.: The reflection of a plane shock wave at a gaseous interface. Proc. Phys. Soc. 61(2), 119-121 (1948)

7. Glass, I.I., Sislian, J.P.: Nonstationary Flows and Shock Waves. Clarendon Press, Oxford (1994)

8. Owczarek, J.A.: Fundamentals of Gas Dynamics. International Textbook Company, Scranton (1964) 